\title{
ANALYSIS OF THE NONCONCENTRIC RADOME-ENCLOSED CYLINDRICAL REFLECTOR ANTENNA SYSTEM, E-POLARIZATION CASE
}

\author{
T. Ŏ̆uzer \\ Department of Electrical and Electronics Engineering \\ Dokuz Eylül University \\ Buca 35160, İzmir, Turkey
}

\author{
A. Altintas \\ Department of Electrical and Electronics Engineering \\ Bilkent University \\ 06533 Ankara, Turkey
}

\begin{abstract}
Two-dimensional (2-D) radiation of a directive complex line source is analyzed in the presence of a perfectly conducting (PEC) reflector antenna system and nonconcentrically located dielectric radome. Similar problem was studied in the literature by using method of regularization and Green's function formulation for the $\mathrm{H}$ polarization case. Here the same techniques are used for $E$-polarization case but in this case the scattered part of the Green's function is computed by using an FFT based algorithm. This provides us to solve the larger geometries accurately in reasonable computer times. So this approach can be considered as another alternative for the analysis of the $E$-polarized radome-enclosed reflector antenna system. Various numerical results are presented to support the convergence and accuracy of the technique and at the same time these results can be considered as reference data.
\end{abstract}

\section{INTRODUCTION}

The radome is a coverage of the reflector antenna. The purpose is to protect the included electronic system from the environmental conditions [1]. However this covering affects the radiation characteristics of the antenna system. Therefore it is necessary to make a proper design of the radome to reduce its negative effects on the radiation 
performance of the reflector antenna. Practically, the reflector antenna system and its radome have 3D geometry but still some 2D reflector-inradome systems are being used in modeling and analysis due to simpler nonvectorial nature. The directive source is commonly modeled by a complex point source [2]. One of the earliest methods to analyze the effect of the radome is the plane-wave spectrum surface integration technique [3]. In that method, the aperture field distribution is considered as a series of rays that are traced through the radome wall and during this transmission the radome wall is modelled by using the locally planar slab geometry. Then the ray tracing was extended by considering the all hybrid ray combinations [4]. In [5] and [6], this extended ray tracing technique was applied to analyze the circular cylindrical radome and the curvature effects were included into the solution. In [7], the beam transmission through $2 \mathrm{D}$ radome is considered by using the complex ray tracing. A different approach is used in [8] by introducing the modal cylindrical-wave spectrum analysis instead of plane wave spectrum. This is one of the earliest methods using cylindrical wave functions and by this way the curvature of the radome was modelled better than the planar slab approximation. Furthermore, it can be said that in all these approaches multiple interactions between the source and radome are ignored. Radome problems can also be solved by using numerical methods like the method of moments [9] or the finite element technique [10]. However these are mainly limited to the electrically small and medium size geometries.

The radiation characteristics of the reflector antenna and radome system can also be analyzed by the method of regularization (MoR) $[11,12]$. A free space analysis of the $2 \mathrm{D}$ reflector antenna system is performed by combining the MoR technique with the complex source point (CSP) method in [13]. Previously the 2D cylindrical reflector antenna was simulated by combining the high frequency ray techniques (AI, UTD) and CSP method in [14]. Therefore the work in [13] was a numerically accurate solution of the same problem. The circularly cylindrical reflector antenna and the covering concentric circular radome was another problem of interest and it was analyzed in [15] for both polarization cases. In all these MoR based solutions, the problem is formulated by considering all requirements of the boundary value problem i.e., a rigorous integral equation approach and therefore taking fully into account all interactions between the elements. In the regularization procedure, the semi-analytical approach is performed. Therefore the operator of the original problem is separated into two parts and the one having the most of the singularity (or preferably the whole singular part) is inverted analytically. The remaining part 
of the original operator is inverted numerically. To perform all these steps, the IE is transformed into the discrete Fourier transform domain and finally the resultant matrix equation appears as Fredholm 2nd kind type so the relative error diminishes as the dimension of the final matrix equation increases. This procedure guarantees that the results converge to the actual solution of the original boundary value problem.

The nonconcentric circular radome covering the circularly cylindrical reflector antenna system is another problem of interest. This configuration is used more frequently in applications to model smaller radomes. In [16], the cylindrical reflector-in-radome antenna system was solved again by combining MoR, Green's function technique and CSP method. Furthermore in this study, it is observed that the radiation characteristics of the free space reflector antenna system can be improved by the proper selection of the nonconcentric radome parameters i.e the radome thickness, its radius, the reflector location inside the radome and the position of the feed.

Here it is tried to observe the radiation characteristics of the cylindrical reflector with its nonconcentric radome for the $E$ polarization case. It is also assumed that the radome and reflector surfaces have circular profiles. In the previous study in [16], a similar radome-enclosed reflector antenna system was analyzed for $H$-polarization case for the moderately-sized geometries. But here it is studied especially for the E-polarization case and even for the larger dimensional geometries. The size limitation in [16] is due to the numerical evaluation of the successive summations. These summations come from the geometrical transformations of the cylindrical wave functions by the well-known addition theorem. However here due to the periodic nature of the problem one can expand Green's function into the Fourier series on the radome centered coordinate system. Then the scattered part of the Green's function can be easily computed in the other reflector centered coordinate system by using the available FFT algorithm. The FFT based formulations of the electromagnetic scattering problems were also used in literature in conjunction with the MoR techniques [17, 18]. In [19], 2D nonconcentric reflector antennain-radome system was solved by the above defined procedure but this time for the $H$-polarization case so as to simulate the larger dimensional geometries. This way of computation provide us to solve the larger problems in the more reasonable CPU time durations and no further efforts have to be spent on the convergence of the successive series.

In the present study, the regularization of the original IE is performed in the discrete Fourier transform domain by using the Riemann-Hilbert Problem (RHP) technique $[11,12]$. This is done in a 
similar way which is described in the all previous MoR based solutions $[11,12]$. But here fortunately the whole singularity of the original operator i.e., the static part inverted analytically. This procedure can be called the most convenient way of the semi-inversion method and also a final matrix equation is obtained as a regularized solution.

The remainder of this paper is organized as follows. In Section 2 the problem is formulated for $E$-polarized radiation in terms of the full-wave electric field integral equation (EFIE). The IE kernel is determined by the Green's function of the circular dielectric radome. This function can be obtained by the coordinate transformation of the cylindrical functions but here the FFT based numerical computation is used. Then, assuming that the reflector surface has the circular profile, the IE is converted to the series equations and the part containing the whole singularity of the IE is inverted analytically by RHP technique. This yields a regularized matrix equation for the surface current expansion coefficients. In Section 3, some radiation characteristics of the reflector-in-radome system are presented. Section 4 contains conclusions of the presented work. A note should be made that the time dependence is assumed as $e^{-i \omega t}$ and omitted throughout the paper.

\section{FORMULATION}

Geometry of the reflector antenna and its covering radome system is given in detail in Figure 1. This radiating system is presented more clearly with all geometrical parts in Figure 2. Here in Figure 1 there is a 2D circularly cylindrical pec reflector surface with radius " $a$ " and its half angle is $\theta_{a p}$. It is covered by a circular dielectric radome which is characterized by the relative permittivity $\varepsilon_{r}$ and the relative permeability $\mu_{r}$. Also the inner and outer radii of the radome are given as $c$ and $d$, respectively. The center of the radome is shifted from the center of the reflector by a distance $L$. The feed is assumed to be a complex point line source located on the symmetry axis of the given system preferably on the approximate geometrical focus i.e., $r_{0}=a / 2$. In addition the complex position of the line source provides us to determine the beamwidth and the beamdirection of the incident field [2]. The free space radiation from such a CSP electric line source is given by

$$
E_{z}^{\text {in }}(\vec{r})=H_{0}^{(1)}\left(k\left|\vec{r}-\vec{r}_{s}\right|\right)
$$

where $k$ is the free-space wavenumber, $\vec{r}_{s}=\vec{r}_{0}+i \vec{b}$ is the complex source position, $\vec{r}_{0}$ is the real-space source position and $\vec{b}$ is the beam parameter vector. The $\vec{r}_{s}, \vec{r}_{0}$ and $\vec{b}$ can be given as $\vec{r}_{s}=\left(r_{s}, \varphi_{s}\right)$, 


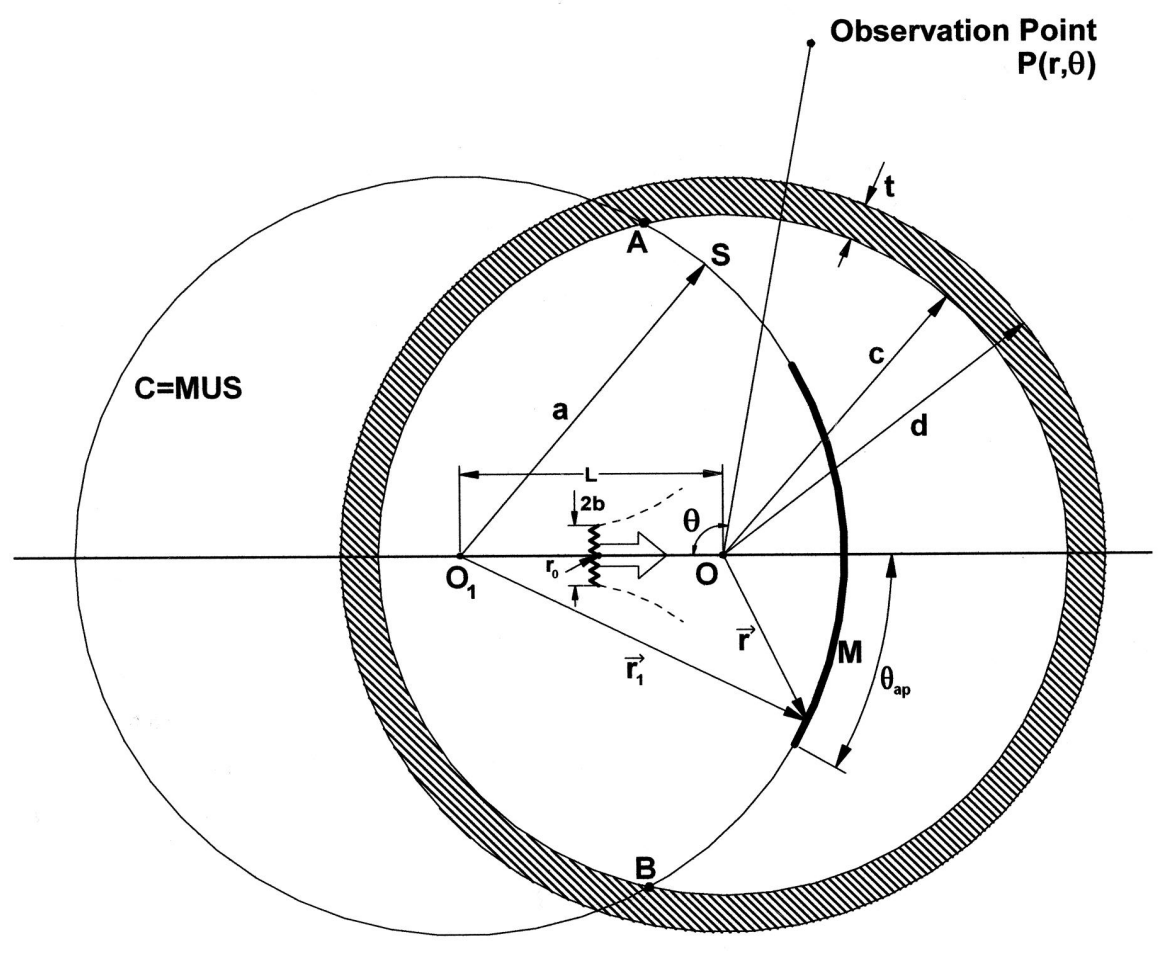

Figure 1. Detailed geometry of the problem with all symbols.

$\vec{r}_{0}=\left(r_{0}, \varphi_{0}\right)$ and $\vec{b}=(b, \beta)$ in polar coordinates. Furthermore $\varphi_{0}$ and $\beta$ can be taken as zero for a symmetric feeding. Assuming that $\operatorname{Re}\left(r_{s}\right)>0$ and $b>0$ one finds that

$$
\begin{aligned}
& r_{s}=\left[r_{0}^{2}+i 2 r_{0} b \cos \left(\beta-\varphi_{0}\right)-b^{2}\right]^{1 / 2} \\
& \varphi_{s}=\arccos \left[\left(r_{0} \cos \varphi_{0}+i b \cos \beta\right) / r_{s}\right]
\end{aligned}
$$

The requirements for the rigorous formulation of the presented problem can be stated as satisfying the 2-D Helmboltz wave equation, the Sommerfeld radiation condition at $r \rightarrow \infty$, the Meixner condition at the reflector edges, the Dirichlet boundary condition on the reflector surface $M$, and further the tangential components of the electric and magnetic field have to be continuous on the radome boundaries.

The following IE is obtained by imposing the Dirichlet boundary 


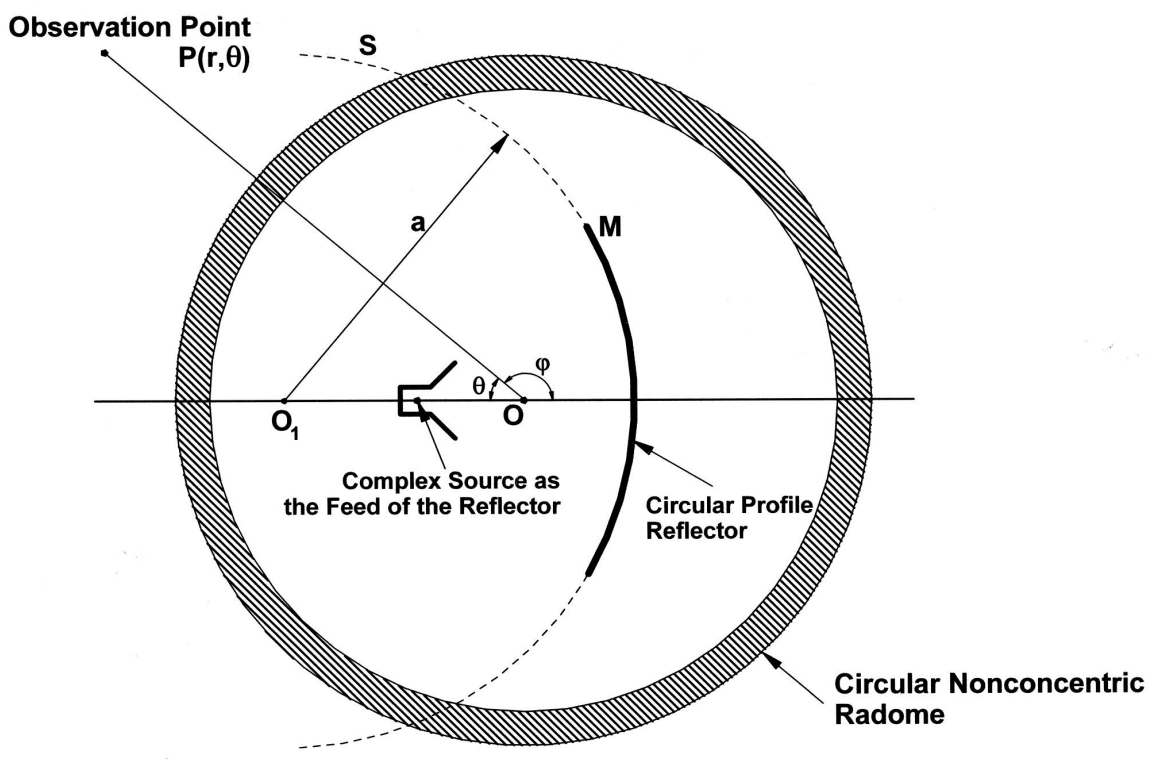

Figure 2. Geometry of the problem in general.

condition on the reflector surface $M$.

$$
\int_{M} J_{z}\left(\vec{r}_{1}^{\prime}\right) G_{z z}^{E}\left(\vec{r}_{1}, \vec{r}_{1}^{\prime}\right) d \vec{r}_{1}^{\prime}=E_{z}^{i n}\left(\vec{r}_{1}^{\prime}\right), \quad \vec{r}_{1} \in M
$$

where $J_{z}$ is the induced surface current density on the reflector surface $M$ and $G_{z z}^{E}$ is the Green's function of the $z$-directed line source in the presence of the circular dielectric shell for the $z$-directed electric field. The function $G_{z z}^{E}$ takes rigorously into account the geometry and material parameters of the radome including the curvature effect, creeping waves, resonances and the radiation condition at $r \rightarrow \infty$. The Green's function $G_{z z}^{E}$ is composed of two parts, i.e., $G_{z z}^{E}=G^{0}+G^{s c}$ where $G^{0}=\frac{i}{4} H_{0}^{(1)}\left(k\left|\vec{r}_{1}-\vec{r}_{1}^{\prime}\right|\right)$ is the free space scalar Green's function in $2 \mathrm{D}$. Besides $G^{S C}$ is the scattered part of the total Green's function of the circular shell which is given explicitly in [15]. In that study the $G^{S C}$ is given in the radome centred $(r, \varphi)$ coordinate system with origin $O$. In order to use it in the Equation (4) it has to be transformed to the $\left(r_{1}, \varphi_{1}\right)$ coordinates. This is performed by using the addition theorem of the cylindrical wave functions in [16]. If this way of formulation is chosen, the successive summations appear in the final result and their 
nature of convergence has to be considered additionally. Alternatively, one can compute the $G^{S C}$ on the closed circular contour $C$ that is the combination of the reflector part $M$ and its complementary part $S$. The expression of the $G^{S C}$ can be given as follow.

$$
\begin{aligned}
G^{s c}\left(\varphi_{1}, \varphi_{1}^{\prime}\right)= & \frac{-i}{4} \sum_{n=-\infty}^{\infty} K_{n}^{e} g\left(r_{1}=a, \varphi_{1}, L\right) g\left(r_{1}^{\prime}=a, \varphi_{1}^{\prime}, L\right) \\
& e^{i n \varphi\left(\varphi_{1}, a, L\right)} e^{-i n \varphi^{\prime}\left(\varphi_{1}^{\prime}, a, L\right)} \quad L<\min (a, c)
\end{aligned}
$$

where $K_{n}^{e}$ is defined in [15]. Additionally this serial expression of the $G^{S C}$ is given detailed in [15] and the radome centred coordinate system is used there. Then the geometrical transformation is performed between two coordinate systems and the $G^{S C}$ is written in terms of the reflector centred coordinates i.e., $\left(r_{1}, \varphi_{1}\right)$ coordinate system.

Here the source and the observation points are taken on $C$ i.e., $r_{1}$ and $r_{1}^{\prime}$ are equal to " $a$ ". Furthermore the $g$ function is defined as follows

$g\left(r_{1}, \varphi_{1}, L\right)=\left\{\begin{array}{l}f\left(r_{1}, \varphi_{1}, L\right) \quad 0 \leq \varphi_{1} \leq \varphi_{m} \text { and } 2 \pi-\varphi_{m} \leq \varphi_{1} \leq 2 \pi \\ \frac{\beta-\alpha}{2\left(\pi-\varphi_{m}\right)} \varphi_{1}+\frac{\alpha\left(2 \pi-\varphi_{m}\right)-\beta \varphi_{m}}{2\left(\pi-\varphi_{m}\right)} \quad \varphi_{m}<\varphi_{1}<2 \pi-\varphi_{m}\end{array}\right.$

where $\alpha=f\left(r_{1}, \varphi_{m}, L\right)$ and $\beta=f\left(r_{1}, 2 \pi-\varphi_{m}, L\right)$. Also the appearing function $f$ is given by $f\left(r_{1}, \varphi_{1}, L\right)=J_{n}\left(k r\left(r_{1}, \varphi_{1}, L\right)\right)$ where $r=\sqrt{r_{1}^{2}+L^{2}-2 r_{1} L \cos \varphi_{1}}$. Also $\varphi\left(\right.$ or $\left.\varphi^{\prime}\right)$ is the function of the $\varphi_{1}$ (or $\varphi^{\prime}$ ) and also related with the other geometrical parameters like $L$ and $a$.

The explicit expression of $G^{S C}$ has the piecewise series form for the three different regions of the radome [15]. These three regions i.e., region 1, region 2 and region 3 are defined as $r<c, c<r<d$ and $r>d$ respectively in the radome centred coordinate system. Here it is used as in the series form of the $G^{S C}(r, \varphi)$ for the region inside the radome i.e., $r, r^{\prime}<c$. For the points outside radome's inner circle i.e., $r, r^{\prime}>c$, the $g\left(r_{1}, \varphi_{1}, L\right)$ and $g\left(r_{1}^{\prime}, \varphi_{1}^{\prime}, L\right)$ functions are defined as an approximation that is the linear equation connecting the end points of the function $f$ from $A\left(a, \varphi_{m}\right)$ to the point $B\left(a, 2 \pi-\varphi_{m}\right)$.

In this way of the definition there is no need to use the all parts of the $G^{S C}$ for the 3 separate regions of the radome. Because it is seen that the only observation and source points on $M$ are crucial in the solution of IE in equation (4). For the other external points on $C$ it may be approximated by any suitable function and here it is chosen as the linear equation between the points $A$ and $B$. 
The mentioned function $G^{S C}\left(\varphi_{1}, \varphi_{1}^{\prime}\right)$ is expanded into the double Fourier series with respect to two arguments producing the coefficients $h_{n m}$. The scattered part of the Green's function i.e., $G^{s c}$ has to be piecewise continuous behaviour on $C$ as indicated in equation (6). So the original function $G^{s c}$ and its first derivative have to exist i.e., piecewise defined and have no singularity. Therefore it can be said that this definition of the $G^{S C}$ on the circular contour $C$ entail that the $h_{n m}$ coefficients asymptotically decay as $O\left(|n|^{-2-\epsilon}|m|^{-2-\epsilon}\right)$, where $\varepsilon$ is any small number i.e., $\varepsilon>0$. Their efficient computation needs double integration of rapidly oscillating functions and here it is performed by the double FFT algorithm. This is the similar to the works which are done in $[17,18]$ for the simulation of the arbitrary profile geometries.

To follow the common procedure of RHP technique given in $[11,12]$ we have to convert the equation (4) to the discrete Fourier transform domain. First of all the current density function is extended by zero value on $S$ and change integration domain in the equation (4) from $M$ to $C$ (i.e., from 0 to $2 \pi$ in $\varphi_{1}^{\prime}$ ). Then the unknown function $J_{z}$ is discretized as follows

$$
J_{z}\left(\varphi_{1}^{\prime}\right)=\sum_{n=-\infty}^{\infty} x_{n} e^{i n \varphi_{1}^{\prime}} \quad \varphi_{1}^{\prime} \in C
$$

Discretization of the equation (4), together with equation $J_{z}\left(\varphi_{1}^{\prime}\right)=0, \varphi_{1}^{\prime} \in S$ leads to the following dual series equations (DSE)

$$
\begin{aligned}
\sum_{n=-\infty}^{\infty} x_{n} Q_{n} e^{i n \varphi_{1}} & =\sum_{n=-\infty}^{\infty} t_{n} e^{i n \varphi_{1}}-\sum_{n=-\infty}^{\infty} b_{n}^{e} e^{i n \varphi_{1}} \varphi_{1} \in M \\
\sum_{n=-\infty}^{\infty} x_{n} e^{i n \varphi_{1}} & =0 \quad \varphi_{1} \in S
\end{aligned}
$$

where $t_{n}=\sum_{p=-\infty}^{\infty} x_{p} h_{n(-p)}-x_{n} K_{n}^{e} J_{n}^{2}(k a)$. Also $Q_{n}$ and $b_{n}^{e}$ are given as $Q_{n}=J_{n}(k a) H_{n}^{(1)}(k a)-K_{n}^{e} J_{n}^{2}(k a)$ and $b_{n}^{e}=J_{n}\left(k r_{s}\right) H_{n}^{(1)}(k a) e^{-i n \varphi_{s}}$ respectively.

By using the new variable $u_{n}=x_{n} Q_{n}-t_{n}+b_{n}^{e}$, one can change the mathematical form of the above DSE into another canonical form given as follows

$$
\begin{aligned}
\sum_{n=-\infty}^{\infty} u_{n}|n| e^{i n \varphi_{1}} & =\sum_{n=-\infty}^{\infty} g_{n} e^{i n \varphi_{1}} \quad \varphi_{1} \in S \\
\sum_{n=-\infty}^{\infty} u_{n} e^{i n \varphi_{1}} & =0 \quad \varphi_{1} \in M
\end{aligned}
$$


where we have denoted.

$$
g_{n}=-u_{n} \Delta_{n}-\frac{1}{i \pi} \frac{t_{n}}{Q_{n}}+\frac{1}{i \pi} \frac{b_{n}^{e}}{Q_{n}} \quad \text { and } \quad \Delta_{n}=\frac{1}{i \pi Q_{n}}-|n|
$$

This canonical DSE is solved analytically in $[11,12]$ and the result is given in [13]. Therefore the resultant matrix equation can be given as the following infinite matrix equation by using the $\tilde{x}_{n}=\frac{x_{n}}{1+|n|}$ transformation

$$
\underbrace{\left(I+A_{m n}^{1}+A_{m n}^{2}+A_{m n}^{3}+A_{m n}^{4}+A_{m n}^{5}\right)}_{A_{m n}} \tilde{x}_{n}=B_{m}^{1}+B_{m}^{2}
$$

where

$$
\begin{aligned}
A_{m n}^{1} & =-(|n|+1) \frac{h_{m(-n)}}{W_{m}} \quad A_{m n}^{2}=\frac{W_{n}}{W_{m}} \Delta_{n} \tilde{T}_{m n} \\
A_{m n}^{3} & =-\frac{(|n|+1)}{W_{m}} \sum_{l=-\infty}^{\infty} h_{l(-n)} \Delta_{l} \tilde{T}_{m l} \\
A_{m n}^{4} & =\frac{1}{i \pi} \frac{(|n|+1)}{W_{m}} \sum_{l=-\infty}^{\infty} \tilde{T}_{m l} \frac{h_{l(-n)}}{Q_{l}} \\
A_{m n}^{5} & =-\frac{i}{\pi} \frac{(|n|+1)}{W_{m}} \frac{K_{n}^{e} J_{n}^{2}(k a)}{Q_{n}} \tilde{T}_{m n} \quad B_{m}^{1}=-\frac{b_{m}^{e}}{W_{m}} \\
B_{m}^{2} & =\sum_{l=-\infty}^{\infty} b_{l}^{e}|l| \frac{\tilde{T}_{m l}}{W_{m}}
\end{aligned}
$$

Also

$$
W_{m}=(|m|+1) J_{m}(k a) H_{m}^{(1)}(k a)
$$

Additionally $\tilde{T}_{m n}=(-1)^{n+m} T_{m n}\left(-\cos \theta_{a p}\right)$ and the $T_{m n}$ functions can be found in $[11,12]$ as combinations of the Legendre polynomials. One can verify that the matrix operators $A_{m n}^{i}$ have bounded norms in $l_{2}$ i.e., $\sum_{n=-\infty}^{\infty} \sum_{m=-\infty}^{\infty}\left|A_{m n}^{i}\right|^{2}<\infty, i=1,2,3,4,5$. Furthermore the right-hand-part elements also belong to $l_{2}$ i.e., $\sum_{m=-\infty}^{\infty}\left|B_{m}^{i}\right|^{2}<\infty, i=1,2$. In this case the infinite matrix Equation (13) is of the Fredholm second kind. Hence the Fredholm theorems guarantee the existence of the unique exact solution $\tilde{x} \in l_{2}$ and also the convergence of the approximate numerical solution when 
truncating the equation (13) with the increasing larger size $N_{t r}$. Furthermore all matrix elements $A_{m n}^{i}$ for $i=1,2,3,4,5$ vanish if $k=0$. Therefore we can say that the resultant regularized matrix equation is obtained by using the static part inversion on the original IE of the problem.

\section{NUMERICAL RESULTS}

The presented formulation has been verified by computing the various relative truncation error plots, radiation patterns, and directivity and total power variations with the problem dimensions. In all these results, the feed antenna is simulated by a CSP method and its beam is characterized by the $k b$ beam width parameter (the beam direction angle is always zero for this symmetrical case). Additionally to generate all these results, we have used a PC notebook Pentium IV , 2.66 GHZ CPU and $256 \mathrm{MB}$ RAM capacity with the windows 2000 operating system.

Depending on the convergent nature of the resultant matrix equation the results mathematically converge to the actual solution of the original problem. However this statement has to be verified by the obtained numerical results. Figure 3 demonstrates the

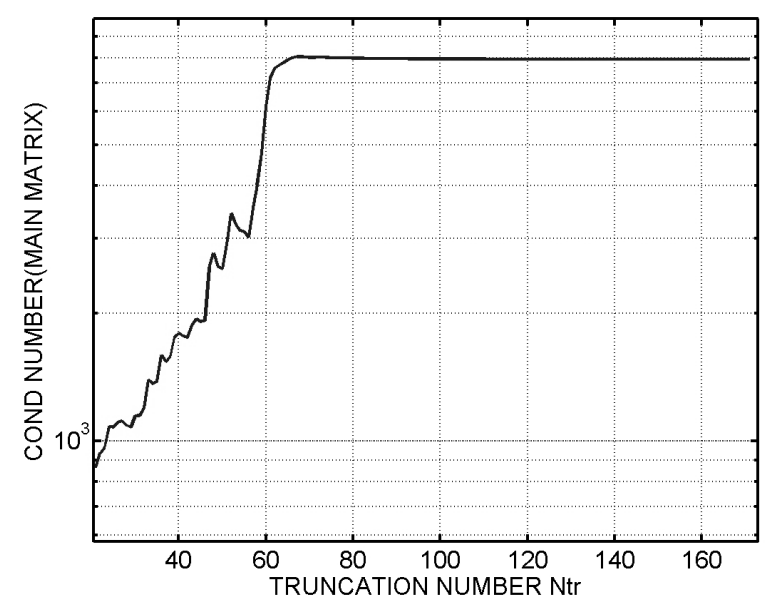

Figure 3. The condition numbers of the MAR matrices i.e., $A_{m n}$ matrices given in the equation (13) versus the truncation number $N_{t r}$. The vertical axis on the plot is in the logarithmic (base 10) scale. The problem parameters are given as $k a=62.8, k L=50, k c=80$, $\theta_{a p}=30^{\circ}, r_{0} / a=0.5, k b=2.6, t=0.4 \lambda_{d}$ and $\varepsilon_{r}=2, \mu_{r}=1$. 
condition number of the main matrix versus the truncation number $N_{t r}$. The main matrix is the square matrix defined in the resultant algebraic matrix equation i.e., $A_{m n}$. It is seen that this value has a rapidly convergent nature with the increasing $N_{t r}$ and this converged level can be considered inside the safety region i.e., not cause to the ill conditioned matrix equation. Besides to verify the actual rate of convergence, we computed the relative error in the obtained surface current density and in the directivity. Here we imply these values in the sense of so-called maximum norm, i.e., $\Delta_{x}=\max \left|x_{n}^{N_{t r}+1}-x_{n}^{N_{t r}}\right| / \max \left|x_{n}^{N_{t r}}\right|$ and also the parameter demonstrating the error in the far field is defined as the relative accuracy in directivity i.e., $\Delta D=\left|D^{N_{t r}+1}-D^{N_{t r}}\right| /\left|D^{N_{t r}}\right|$. Figure 4(a) presents the plot of the directivity versus the truncation number and it is seen that the results really converge sufficiently at a reasonable matrix dimension. This is also obvious from the Figure 4(b) and practically speaking 6-digit relative accuracy in the directivity $(\Delta D)$ is obtained. As expected, this quantity decays faster than the
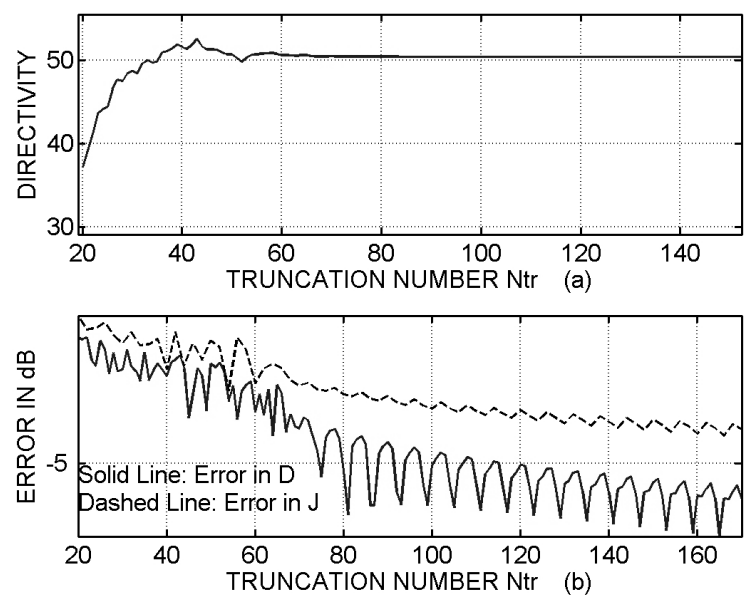

Figure 4. (a) The directivity computed from the presented solution versus the truncation error. (b) The relative accuracy of the surfacecurrent coefficients and the directivity in terms of the truncation number $N_{t r}$ in logarithmic scale. The problem parameters are given as $k a=62.8, k L=50, k c=80, \theta_{a p}=30^{\circ}, r_{0} / a=0.5, k b=2.6$, $t=0.4 \lambda_{d}$ and $\varepsilon_{r}=2, \mu_{r}=1$. 
near-field error that is the relative error in the surface current density. It can also be seen that the 4-digit relative accuracy is obtained in this surface current computation. When computing the $h_{n m}$ coefficients, FFT algorithm was used with the order of $2048 \times 2048$. All of these results support the convergent statement of the presented formulation in the limits of the computer precision.

The running cpu times of the written computer code is presented in Table 1 in two stages i.e., the filling of the matrices and its the solution time and full program running time with the all parameters computation. This table indicates that in addition to the accuracy and convergence, the solution time of the presented formulation is reasonably fast.

Table 1. Running CPU times of the computer code $\Theta_{a p}=30^{\circ}, r_{0} / a=$ $0.5, k b=2.6, \varepsilon_{r}=2, \mu_{r}=1$ and $t=0.4 \lambda_{d}$.

\begin{tabular}{|l|l|l|}
\hline & $\begin{array}{l}\text { Filling of the matrices } \\
\text { and its solution time } \\
\text { (seconds) }\end{array}$ & $\begin{array}{l}\text { Full program running } \\
\text { time (seconds) }\end{array}$ \\
\hline $\begin{array}{l}k a=62.8, k L=50 \text { and } k c=80 \\
N t r=130\end{array}$ & 124.96 & 157.71 \\
\hline $\begin{array}{l}k a=94.2478, k L=75.3982 \\
\text { and } k c=105.5575, N t r=170\end{array}$ & 202.95 & 255.63 \\
\hline $\begin{array}{l}k a=157.0796, k L=125.6037 \\
\text { and } k c=175.9292, N t r=270\end{array}$ & 615.32 & 722.76 \\
\hline
\end{tabular}

Another parameter of interest is the normalized radiation pattern of the defined reflector antenna system with its covering radome. Figure 5 presents the radiation pattern of a reflector with the $25 \lambda$ aperture dimension. In this figure the thickness of the radome is taken at first as the $0.4 \lambda_{d}$ and then the half wavelength case is also plotted with the free space case on the same figure. It is seen that the half wavelength design appreciably prevents the distortions on the pattern and it becomes very close to the free space radiation of the reflector antenna system. The distortions on the pattern for the $0.4 \lambda_{d}$ is mainly appear on the penumbra region and on the spillover lobe after the peak level towards the back side region. These may be explained by considering the possible reflections of the radiation of the reflector antenna system from the circular radome. The oscillation in the radiation pattern increases especially for the penumbra and back side lobes region if the aperture dimension increases but if the feed 


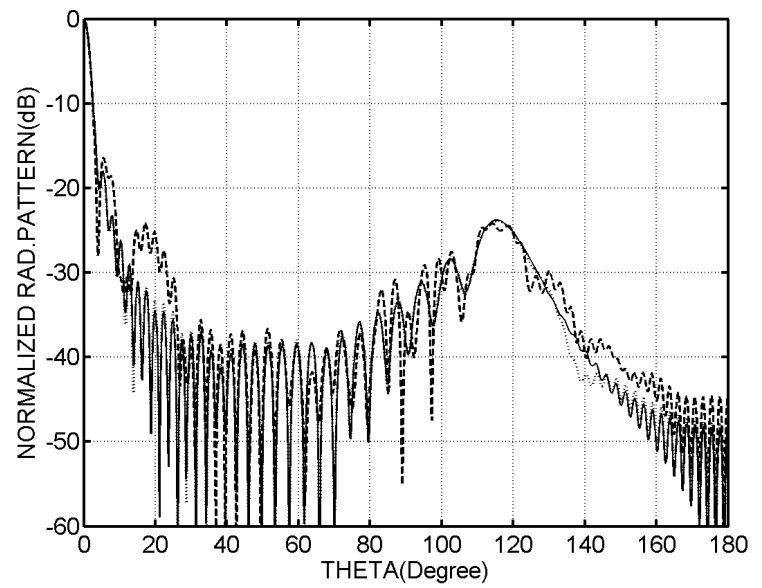

Figure 5. The comparison of the normalized radiation patterns for the different radome thickness and for the free space case. The problem dimensions are given as $k a=157.0796, k L=125.6637, k c=175.9292$, $\theta_{a p}=30^{\circ}, r_{0} / a=0.5, k b=2.6$, and $\varepsilon_{r}=2, \mu_{r}=1$. Solid line: free space solution, dashed line: $t=0.4 \lambda_{d}$ and dotted line: $t=0.5 \lambda_{d}$.
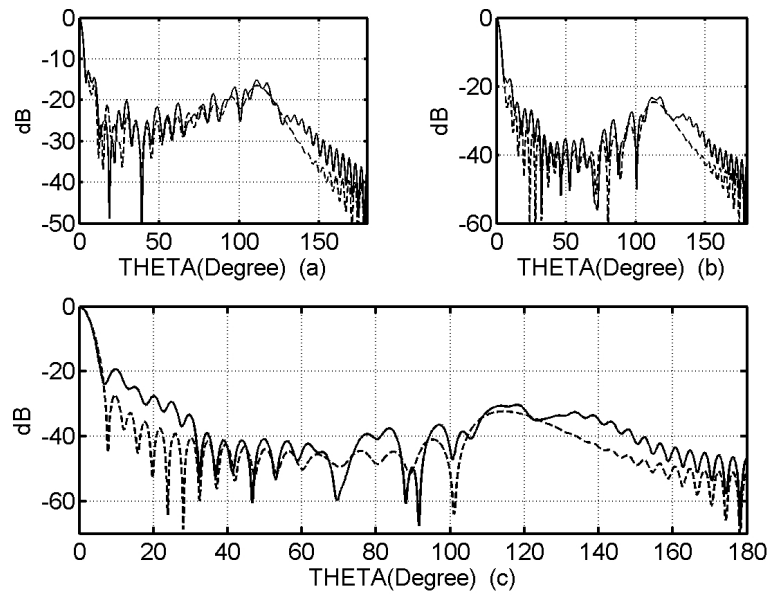

Figure 6. Normalized radiation patterns are given for the free space and for the $t=0.4 \lambda_{d}$ cases and the problem parameters are given as $k a=94.2478, k L=75.3982, k c=105.5575, \theta_{a p}=30^{\circ}, r_{0} / a=0.5$, and $\varepsilon_{r}=2, \mu_{r}=1$. (a) Source directivity parameters $(k b)$ is taken as 1 (b) Source directivity parameters $(k b)$ is taken as 3 (c) Source directivity parameters $(k b)$ is taken as 5 . In all three cases the solid line represents $t=0.4 \lambda_{d}$ radome thickness and the dashed line represents the free space case. 
illumination is constant then spillover lobe level will not be changed so much.

Figure 6 presents the normalized radiation patterns for different source directivity parameter $k b$. In the same figure the radome thickness is taken as $t=0.4 \lambda$ and the free space case is also plotted in each case. It is seen that the free space radiation levels drop in each region in the radiation patterns with the increasing $k b$ values. This is an obvious fact because the edges are illuminated less and less due to the more directive primary feed pattern. On the other hand, the deviation of the radiation pattern for the $t=0.4 \lambda_{d}$ case from the free space geometry increases especially in the early penumbra region but the distortion remains in the same level in the spillover lobe towards to the back side region. This may be due to the higher directive primary feed causes the propagation of the wider beam through the radome.

In Figures 7 and 8, the variation of the directivity and the normalized power with the radome thickness and the relative position $r_{0} / a$ is presented. Also in these computations the optimization procedure is considered by following the similar study performed in [16] and the almost in the same level and type of the optimization is observed again for this $E$-polarization case. Figure 7(a) shows that the directivity versus $r_{0} / a$ variation for the different radome thicknesses

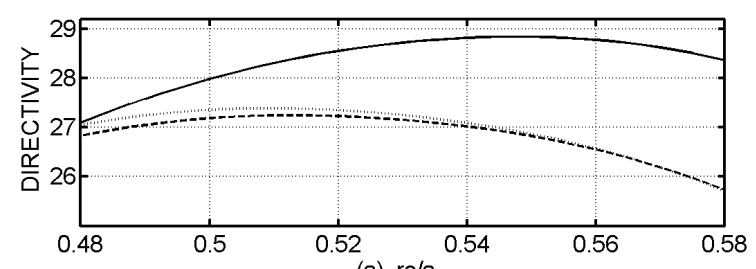

(a) ro/a

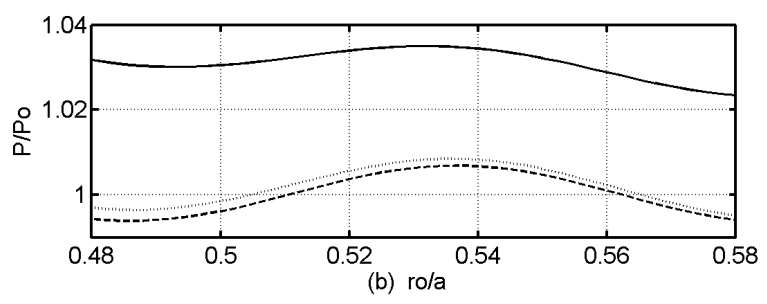

Figure 7. Directivity versus feed position defined as $r_{0} / a$. (b) Ratio of the total radiated power $P$ to the incident power $P_{0}$ i.e., $P / P_{0}$ versus feed position $r_{0} / a$. Problem parameters are given as $k a=31.4$, $k L=26.95, k c=37.69, \theta_{a p}=30^{\circ}, k b=2.6, \varepsilon_{r}=2, \mu_{r}=1$. In two cases solid line: $t=0.375 \lambda_{d}$, dashed line: free space solution and dotted line: $t=0.5 \lambda_{d}$. 

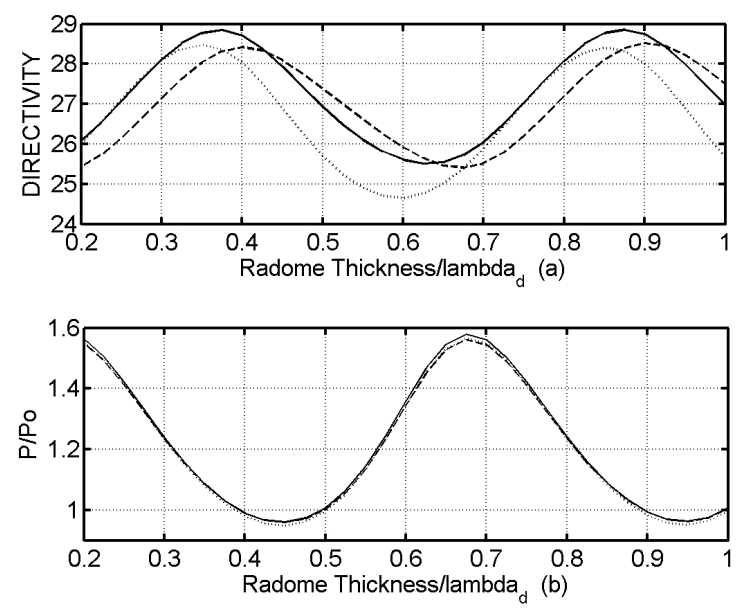

Figure 8. (a) Directivity versus radome thickness in terms of wavelength in radome. (b) Ratio of the total radiated power $P$ to the incident power $P_{0}$ i.e., $P / P_{0}$ versus radome thickness in terms of wavelength in radome. Problem parameters are given as $k a=31.4$, $k L=26.95, k c=37.69, \theta_{a p}=30^{\circ}, k b=2.6, \varepsilon_{r}=2, \mu_{r}=1$. Plots are obtained for 3 different $r_{0} / a$ values as solid line: $r_{0} / a=0.5475$, dashed line: $r_{0} / a=0.51$ and dotted line: $r_{0} / a=0.58$.

and it is seen that the higher directivity values can be obtained for the $t=0.375 \lambda_{d}$ case. In this optimized curve, the maximum is obtained for the relative feed position i.e., $r_{0} / a=0.5475$. The level of increase in directivity is not so much but it is compatible with the previous $H$-pol case given in [16]. Figure 7(b) presents the normalized power variation with the relative position $r_{0} / a$. It is observed that the higher directivity curve has also higher normalized power levels. In the next plot Figure 8(a) if one takes the relative feed position as the $r_{0} / a=0.5475$ then the directivity versus the radome thickness has an optimum value at $t=0.375 \lambda_{d}$. This is the same optimum point also indicated in Figure 7. Figure 8(b) shows the normalized power variation with respect to the radome thickness and all curves almost in a same range of variation.

Figures 9 and 10 present the directivity and the normalized power variation similarly obtained in the previous figures but this time for the approximately three times larger than the geometry used in Figures 7 and 8. Firstly it is seen in Figure 9(a) that the directivity has an optimum value around the $r_{0} / a=0.523$ for both free space and $t=0.5 \lambda_{d}$ cases. Additionally two curves are almost matched for 

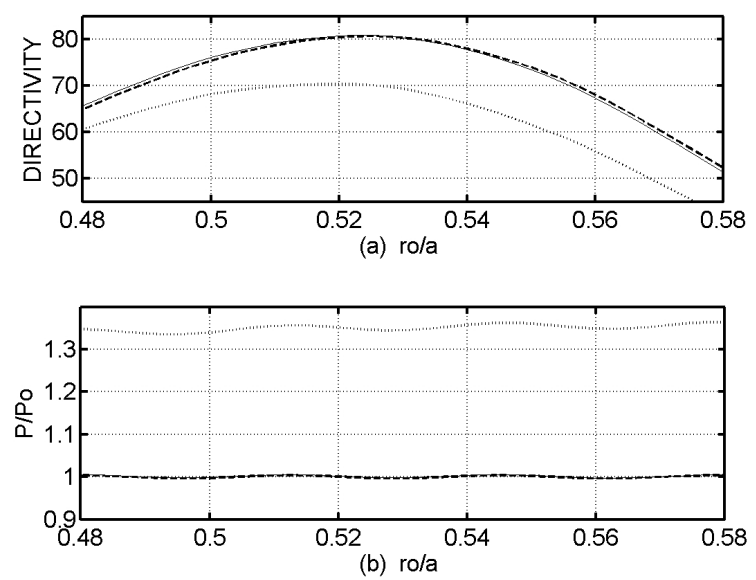

Figure 9. (a) Directivity versus feed position defined as $r_{0} / a$. (b) Ratio of the total radiated power $P$ to the incident power $P_{0}$ i.e., $P / P_{0}$ versus feed position $r_{0} / a$. Problem parameters are given as $k a=94.2$, $k L=80.85, k c=113.07, \theta_{a p}=30^{\circ}, k b=2.6, \varepsilon_{r}=2, \mu_{r}=1$. In two cases solid line: $t=0.5 \lambda_{d}$, dashed line: free space and dotted line: $t=0.26 \lambda_{d}$.
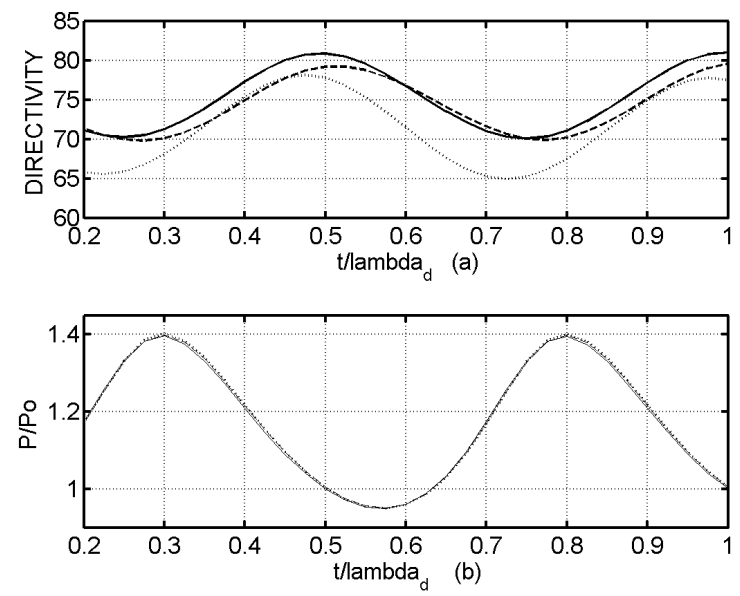

Figure 10. (a) Directivity versus radome thickness in terms of wavelength in radome. (b) Ratio of the total radiated power $P$ to the incident power $P_{0}$ i.e., $P / P_{0}$ versus radome thickness in terms of wavelength in radome. Problem parameters are given as $k a=94.2$, $k L=80.85, k c=113.07, \theta_{a p}=30^{\circ}, k b=2.6, \varepsilon_{r}=2, \mu_{r}=1$. Solid line: $r_{0} / a=0.523$, dashed line: $r_{0} / a=0.51$ and dotted line: $r_{0} / a=0.54$. 
these two situations. If the radome thickness is $t=0.26 \lambda_{d}$ then the directivity versus $r_{0} / a$ curve is lower. In Figure 9(b) the normalized power variations are observed and the lower directivity case now presents the higher power behavior. Secondly Figure 10 is closely related with the Figure 8 and in this case the variations occur with the radome thickness. By considering the half wavelength design criteria, one can expect that $t=0.5 \lambda_{d}$ case the directivity and pattern will be approaching to the free space case (no radome case) and it is already so that. Figure 10(a) shows that the directivity has an optimum value around $t=0.5 \lambda_{d}$. For this radome thickness point i.e., $t=0.5 \lambda_{d}$ the maximum directivity is observed at the $r_{0} / a=0.523$ and almost in the same level with the free space case. Figure 10(b) shows the normalized power variation with respect to the radome thickness and all curves almost in a same range of variation.

So one can say that for this larger geometry the design of the radome shape does not provide an reasonable increase in the directivity of the overall reflector antenna and the radome system.

\section{CONCLUSION}

2-D circularly reflector antenna with its nonconcentric radome has been modelled by the CSP-RHP approach for the E-polarization case. This is the continuation of the similar study performed for the $\mathrm{H}$ polarization in [16]. In the formulation FFT based algorithm is used in the computation of the Green's function. This enables us to analyze electrically larger geometries. The plots of the computational error versus the truncation number support the convergence statement. Radiation characteristics of the studied system have been examined by computing the various radiation patterns, directivity and the normalized power plots. Presented results justify the basic conclusions of the practical antenna engineering.

\section{ACKNOWLEDGMENT}

The authors would like to thank Prof. Dr. Alexander I. Nosich for his useful suggestions and support.

\section{REFERENCES}

1. Kraus, J. D. and R. J. Marhefka, Antennas for All Applications, Mc Graw Hill, 2002.

2. Felsen, L. B., "Complex source point solutions of the field equations and their relation to the propagating and scattering of 
Gaussian beams," Symp. Math., Vol. 18, 39-56, Academic Press, NY, 1976.

3. Wu, D. C. F. and R. C. Rudduck, "Plane wave spectrum surface integration technique analysis," IEEE Trans. Antennas and Propagation, Vol. AP-22, 497-500, May 1974.

4. Einziger, P. D. and L. B. Felsen, "Ray analysis of two-dimensional radomes," IEEE Trans. Antennas and Propagation, Vol. AP-31, No. 6, 870-884, November 1983.

5. Einziger, P. D. and L. B. Felsen, "Rigorous asymptotic analysis of transmission through a curved dielectric slab," IEEE Trans. Antennas and Propagation, Vol. AP-31, No. 6, 863-870, November 1983.

6. Felsen, L. B., N. Subramaniam, and K. Arichandran, "Equivalence relation between partial angular harmonic and ray-type green's functions for a cylindrical dielectric layer," IEEE Trans. Antennas and Propagation, Vol. AP-38, No. 8, 1273-1279, August 1990.

7. Gao, X. J. and L. B. Felsen, "Complex ray analysis of beam transmission through two-dimensional radomes," IEEE Trans. Antennas and Propagation, Vol. AP-33, No. 9, 963-975, September 1985.

8. Chang, J.-H. and K.-K. Chan, "Analysis of a two-dimensional radome of arbitrarily curved surface," IEEE Trans. Antennas and Propagation, Vol. AP-38, No. 10, 165-1568, October 1990.

9. Arvas, E. and S. Ponnopalli, "Scattering cross section of a small radome of arbitrary shape," IEEE Trans. Antennas and Propagation, Vol. AP-37, May 1989.

10. Gordon, R. K. and R. Mittra, "Finite element analysis of axisymmetric radomes," IEEE Trans. Antennas and Propagation," Vol. AP-41, 975-980, 1993.

11. Nosich, A. I., "MAR in the wave-scattering and eigenvalue problems: foundations and review of solutions," IEEE Antennas Propagat. Magazine, Vol. 42, No. 3, 34-49, 1999.

12. Nosich, A. I., "Green's function - dual series approach in wave scattering from combined resonant scatterers," Analytical and Numerical Methods in Electromagnetic Wave Theory, M. Hashimoto et al. (Eds.), 419-469, Science House, Tokyo, 1993.

13. Oğuzer, T., A. Altintas, and A. I. Nosich, "Accurate simulation of reflector antennas by the complex source - dual series approach," IEEE Trans. Antennas Propagat., Vol. AP-43, No. 8, 793-802, 1995.

14. Suedan, G. A. and E. V. Jull, "Beam diffraction by planar and 
parabolic reflectors," IEEE Trans. Antennas Propagat., Vol. AP39, No. 4, 521-527, 1991.

15. Oğuzer, T., "Analysis of circular reflector antenna covered by concentric dielectric radome," IEEE Trans. Antennas Propagat., Vol. AP-49, No. 3, 458-464, March 2001.

16. Yurchenko, V. B., A. Altintas, and A. I. Nosich, "Numerical optimization of a cylindrical reflector-in-radome antenna system," IEEE Trans. Antennas Propagat., Vol. AP-47, No. 4, 668-673, 1999.

17. Ŏguzer, T., A. I. Nosich, and A. Altintas, "E-polarized beam scattering by an open cylindrical PEC strip having arbitrary conical-section profile," Microwave Optical Technology Letters, Vol. 31, No. 6, 480-484, 2001.

18. Nosich, A. I. and S. V. Boriskina, "MAR in the problems of wave diffraction by dielectric cylinders of arbitrary cross-sections," Radio Physics and Radio Astronomy, Vol. 3, No. 4, 405-413, 1998.

19. Oğuzer, T., "Analysis of the $2 \mathrm{D}$ nonconcentric large reflector antenna-in-radome system: H-polarized case," Mathematical Methods in Electromagnetic Theory MMET2004 International Symposium Proceedings, 415-417. 